\title{
Study on the Factors Affecting the Psychological Fatigue of the Dispatching Workers
}

\author{
Rouling $\mathrm{He}^{\mathrm{a},{ }^{*}}$ \\ School of Traffic and Transportation, Beijing JiaoTong University, Beijing 10044, China \\ *, a 16120812@bjtu.edu.cn
}

Keywords: Psychological fatigue; fatigue determination; dispatch; influence factors.

\begin{abstract}
The core work of railway and rail transportation is the dispatching work. Long-time repetitive dispatching work is very easy to cause fatigue, burnout and other psychological state of the employees. This paper focuses on the psychological fatigue caused by dispatching work, using the professional fatigue measurement scale to eliminate interference factors, and using fatigue testing software to study the influencing factors of dispatching fatigue. After testing the fatigue, the conclusion can be obtained from the comparative analysis: Increase in dispatching work time leads to deepening the degree of psychological fatigue; Women are more capable of fighting psychological fatigue; working experience helps to attract attention.
\end{abstract}

\section{Introduction}

Railway, rail transit and other transport modes play important roles in the national economic life. Among them, the dispatching is at the core. The dispatch needs a high concentration of spirit, the long period of work produces fatigue, affecting the efficiency of the work.

According to the different mechanism of fatigue, it can be divided into two kinds of phychological fatigue and psychological fatigue. In view of fatigue, foreign scholars and domestic academics have made a thorough study from various angles and different emphasis. RADUM[1]thought that the driver's gender, age, and other demographic factors have certain influence on the fatigue; RONG's research indicates that the sympathetic nerve activity and the resting [2] of the parasympathetic nerve can occur in the ECG signals caused by fatigue. The study of MORRIS shows that the equilibrium state of sympathetic parasympathetic tension can be used as an index for fatigue detection[3]; The fatigue driving test model is established by $\mathrm{Xu}$ Chuan considering individual differences, which improves the fatigue monitoring precision [4]. Guo Zizheng and so on divided the fatigue grade by the time period, and built the driver's fatigue state recognition model combining the support vector mechanism [5]. At present, great progress has been made in the research and detection technology of fatigue caused by [6].

In conclusion, domestic and foreign scholars have qualitatively and quantitatively analyzed the causes and detection methods of fatigue from different angles, and put forward the measures to mitigate fatigue in many aspects. However, the factors affecting the fatigue of the dispatcher have not been studied. Because of the particularity of the dispatcher's work, the working efficiency of the employees directly affects the efficiency of the whole station (sites) and even the whole railway network. With The simple scheduling process and scattered spare time, how to determine the influencing factors of the dispatchers' fatigue, reduce the fatigue, and improve the efficiency of dispatchers is the main problem to be solved in this paper.

The common fatigue testing methods, such as the measurement of the knee tendon reflex threshold and the skin current reaction, have some limitations in accuracy. The fatigue testing software used in this study is mainly determined by identifying the average reaction time and error rate of the specified graphics. 


\section{Experimental Determination}

\subsection{Test Tools}

(1) Fatigue measurement scale

Fatigue scale -14 (Fatigue Scale-14 FS-14) is composed by many experts--Trudie Chalder from Psychosocial research office in King's College Hospital and G. Berelowitz in Queen Mary's University Hospital, in order to eliminate the negative impact of the experimenter.

(2) Comprehensive dispatching system for urban rail transit (Figure 1)
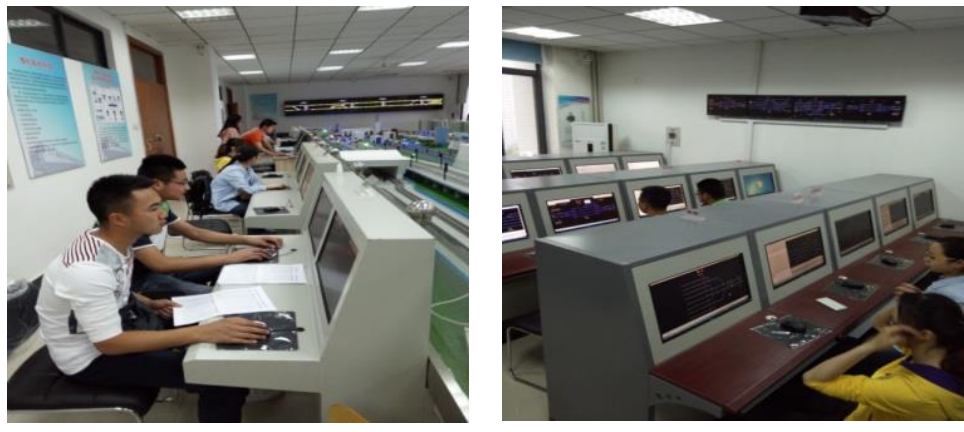

Figure 1 Experimental scene

Comprehensive Laboratory of urban rail transit of the college of transportation engineering department of China University of Mining and Technology is just putting into use .The teaching equipment can fully meet the demand of simulation training of the students for the railway, urban rail transit and scheduling, and the sand table can be used, which provide good conditions for the experiment.

(3) Fatigue testing software
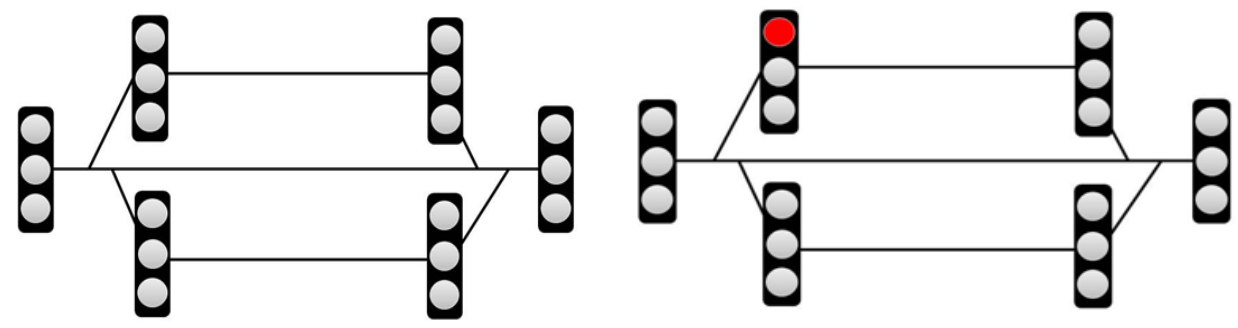

Figure 2 The main interface of fatigue testing software

The master interface of the test software is shown in Figure 2. The test prompt is "the mass transit fatigue test, please quickly click on the red light"; With timing start, the first upper part of each signal lamp lights turn red randomly (i.e. red circle covers the original gray circle, as shown in Figure 2), the red circle appeared in a total of 200 (random position).

\subsection{Experimental Process}

(1)The testers should fill out the fatigue assessment scale to record the fatigue state of the tested person and exclude the influence of other factors.

(2)The subjects began to simulate the scheduling work, with or without sand model in the site, Eprime mental fatigue test, started before the scheduling, was operated normally for 15 minutes, and started again after 15 minutes, then carried out the dispatching work, and performed the E-prime mental fatigue test, in a total of 5 times.

(3)On the first day, the experiment was conducted by 1,2, 3 and 4 experimenters. They completed two groups of experiments without sand table (R1) and with sand table (R2). Number of 1, 2, 3, 4, 5, 6, 7 and 8 experimenters were tested during DAY1 experiments respectively. They completed two groups of experiments without sand table and with sand table. The experimental process, as shown in Figure 3, described the basic situation of the DAY1 and DAY2 experimenters. 


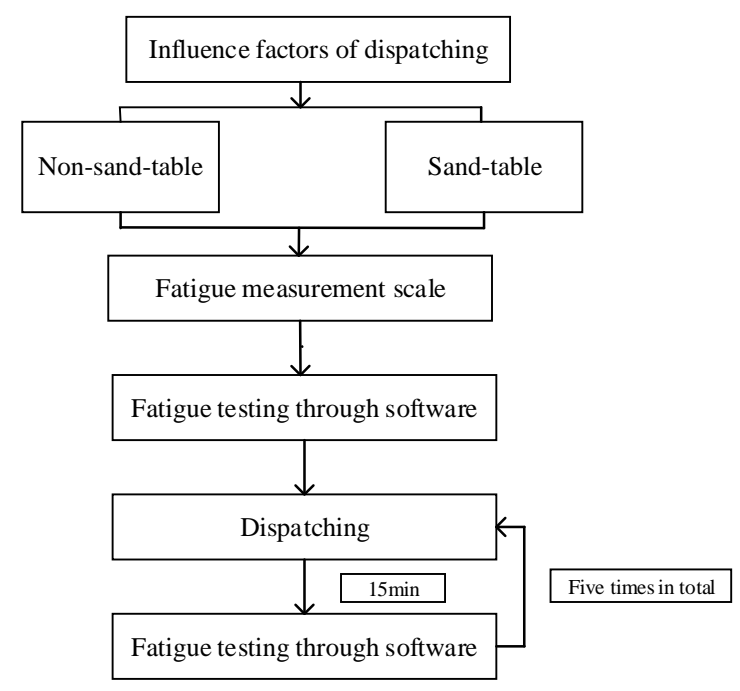

Figure 3 Experimental process

Table 1 Experimental arrangement

\begin{tabular}{cccccc}
\hline Experimenter & Gender & \multicolumn{2}{c}{ DAY1 } & \multicolumn{2}{c}{ DAY2 } \\
& & R1 & R2 & R1 & R2 \\
\hline NO. 1 & M & $\sqrt{ }$ & $\sqrt{ }$ & $\sqrt{ }$ & $\sqrt{ }$ \\
NO.2 & M & $\sqrt{ }$ & $\sqrt{ }$ & $\sqrt{ }$ & $\sqrt{ }$ \\
NO.3 & F & $\sqrt{ }$ & $\sqrt{ }$ & $\sqrt{ }$ & $\sqrt{ }$ \\
NO. 4 & F & $\sqrt{ }$ & $\sqrt{ }$ & $\sqrt{ }$ & $\sqrt{ }$ \\
NO.5 & M & $\times$ & $\times$ & $\sqrt{ }$ & $\sqrt{ }$ \\
NO.6 & M & $\times$ & $\times$ & $\sqrt{ }$ & $\sqrt{ }$ \\
NO.7 & F & $\times$ & $\times$ & $\sqrt{ }$ & $\sqrt{ }$ \\
NO.8 & F & $\times$ & $\times$ & $\sqrt{ }$ & $\sqrt{ }$ \\
\hline
\end{tabular}

\section{Data Results and Analysis}

The experiment involves several factors, and the analysis of the experimental data is carried out in the case of the control variables.

\subsection{Effect of Time on Scheduling Fatigue}

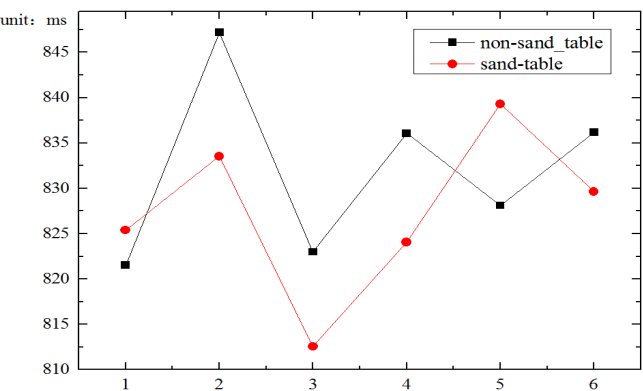

(a)

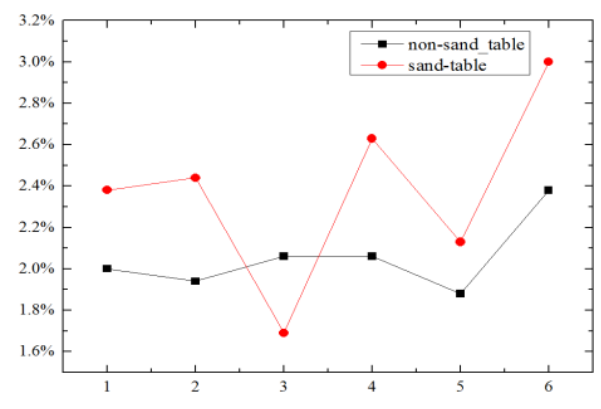

(b)

Figure 4 Reaction time and percentage of errors.

Figure 4(a) is the varying reaction time with dispatching time and the right one is the changing percentage of errors and Figure 4(b) is corresponding percentage of errors in fatigue testing.

It can be concluded that when the experimenters were engaged in scheduling and driving work, the fatigue degree increased with the increase of working hours, which showed the slower response rate and higher click error rate of the fatigue test. The fatigue test data for dispatching work without sand table or with sand table was analyzed, and showed that: as time went by, the average reaction 
time slightly increased, and the fatigue of experimenter was gradually deepened, the percentage of errors fluctuated, which indicated the experimenters were trying to adjust their working state during the experiment.

\subsection{Effect of Gender on Scheduling Fatigue}

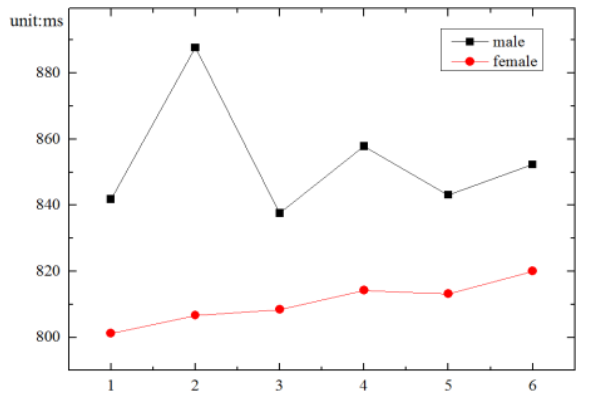

(a)

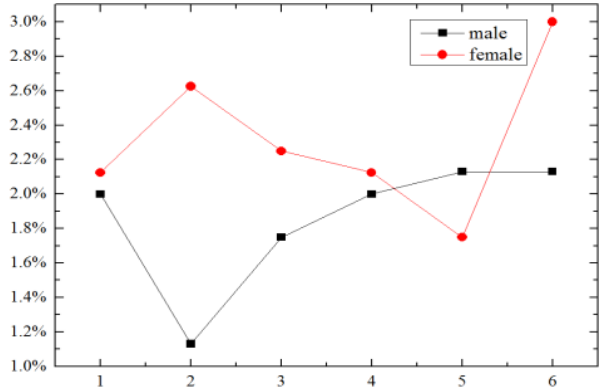

(b)

Figure 5 Reaction time and percentage of errors.

Figure 5(a) is the varying reaction time and Figure 5(b) is corresponding percentage of errors in fatigue testing between male and female. In comparison with all cases, female have stronger ability to resist fatigue in dispatching work, mainly in the following aspects: the average reaction time is shorter than that of male, which can guarantee higher accuracy at faster reaction speed.

Women are easily disturbed by changes in the external environment, reducing the correct rate of work, but women adapt to the cases faster, and can soon be restored to normal state; Men were less disturbed by the external environment, but the average reaction time of men was slightly higher comparing in the absence of sand table with in the presence of sand table, but not much.

\subsection{Effect of Experience on Scheduling Fatigue}
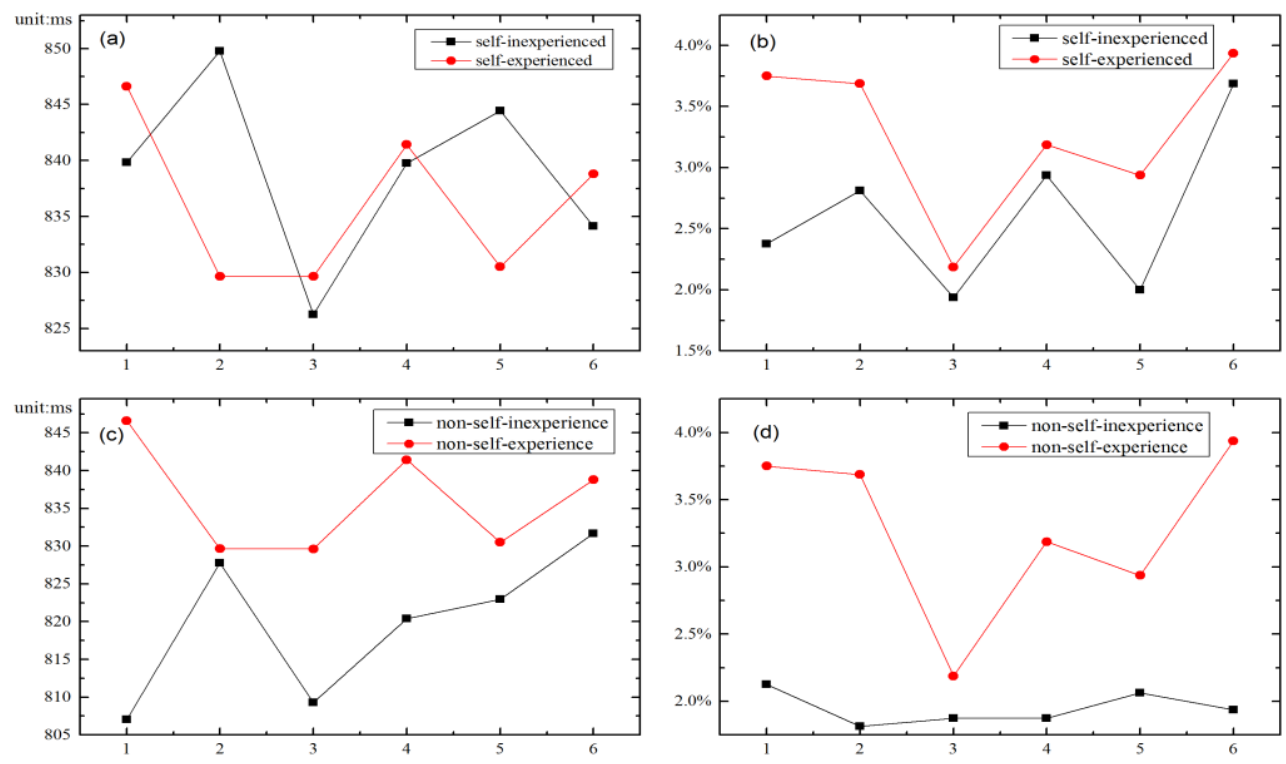

Figure 6 Reaction time and percentage of errors

Figure 6(a) shows the contrast of the experimenter's own different states while Figure 6(C) compares experienced experimenters with inexperienced ones. Figure 6(b) and Figure 6(d) are corresponding percentage of errors. There was twice fluctuation of average reaction time when they were inexperienced. The peak value of the two fluctuation corresponds to the lower value of experience, while the average error rate of the whole group without experience is less than with experience. As a result, the experience has made the experimenter more focused on its own reaction speed and attention.

According to the analysis of non-self-experience parameters, the average response time and the average error rate of the experienced experimenters were higher than those of the inexperienced experimenters, which indicated that the sufficient experience make experimenters exhausted. 


\section{Conclusion}

This study is based on three factors, time, gender and empirical parameters. The fatigue test is carried out in two cases without sand table and with sand table at the same time. According to the obtained data, the sand table has no effect on the dispatcher's fatigue, which means in the case of remote scheduling and on-site visibility, there is no difference in the fatigue of the dispatcher. With the increase of scheduling work time, the experimenter's fatigue is gradually deepened, at the same time, the experimenter has a certain adjustment ability. Women have better performance in fatigue experiments, that is, women's ability to resist fatigue is stronger. Self-experience improves attention, but in the whole, sufficient experience makes people tired of work.

\section{References}

[1]. RADUM I, RADUN J, WAHDE M, et al. Self-reported circumstances and consequences of driving while sleepy [J]. Transportation Research Part F Traffic Psychology \& Behavior, 2015, 32:91-100.

[2]. RONG J. Sound as Countermeasure against Driving Fatigue Based on ECG[C]// ICCTP 2020@sIntegrated Transportation Systems: Green, Intelligent, Reliable. ASCE, 2015.

[3]. MORRIS D M, PILCHER J J, III F S S. Lane heading difference: An innovative model for drowsy driving detection using retrospective analysis around curves [J]. Accident Analysis \& Prevention,2015, 80:117-124

[4]. XU Chuan, PEI Sai-jun, WANG Xue-song. Driver Drowsiness Detection Based on Nonintrusive Metrics Considering Individual Difference [J]. China Journal of Highway and Transport, 2016, 29(10):118-125.

[5]. GUO Zizheng, NIU Linbo, WU Zhimin, XIAO Qiong, SHI Lei. Driver's Fatigue Recognition Algorithm Based on EEG and Its Validity Verification [J]. JOURNAL OF BEIJING UNIVERISITY OF TECHNOLOGY, 2017, 43(06): 929-934.

[6]. XIAO Sai, LEI Yewei. Research on the causes for driver fatigue and the monitoring technology progress [J]. Technology \& Economy in Areas of Communications, 2017, 19(04):14-19+63. 